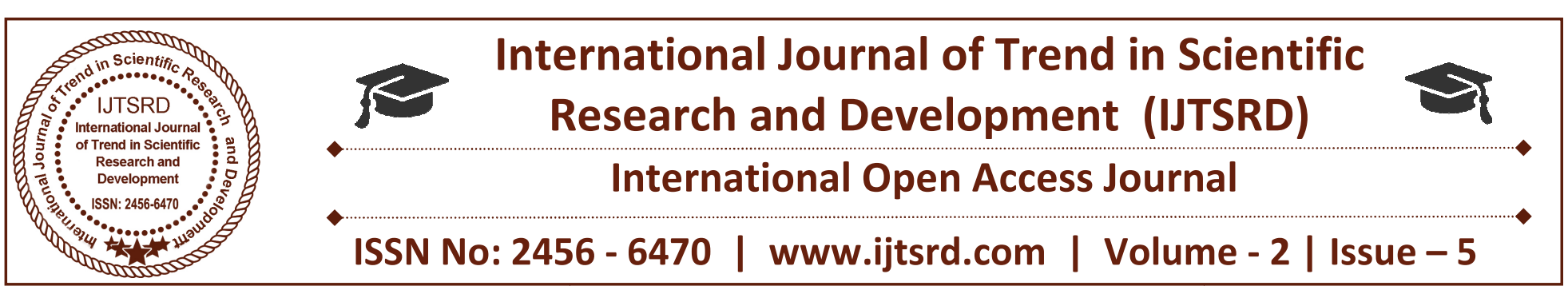

\title{
Face Recognition by using Wavelet Based Frame Work
}

\author{
Dr. Aziz Makandar ${ }^{1}$, Mrs. Rashmi Somshekhar ${ }^{2}$, Ms. Smitha $\mathbf{M}^{3}$ \\ ${ }^{1}$ Professor, ${ }^{2}$ Research Scholar, ${ }^{3}$ Student \\ Department of Computer Science, Akkamahadevi Women's University, \\ Vijayapura, Karnataka, India
}

\begin{abstract}
Content-based indexing methods are of great interest for image and video retrieval in audio-visual archives, such as in the DiVAN project that we are currently developing. Detecting and recognizing human faces automatically in video data provide users with powerful tools for performing queries. The work is done for recognition of the face by using wavelet packet decomposition. Each face is described by a subset of band filtered images containing wavelet coefficients. These coefficients characterize the face texture and a set of simple statistical measures allow us to form compact and meaningful feature vectors. An efficient and reliable probalistic metric derived from the Bhattacharyya distance is used in order to classify the face feature vectors into person classes.
\end{abstract}

Keywords: Face Recognition, Wavelet packet decomposition, Bhattacharyya distance

\section{INTRODUCTION}

Face recognition is becoming a very promising tool for automatic multimedia content analysis and for content based indexing video retrieval system. Such a system is currently developed within the Esprit project DiVAN ([5]) which aims at building and evaluating a distributed audio-visual archives network providing a community of users with facilities to store video raw material, and access it in a coherent way, on top of high-speed wide area communication networks. The video raw data is first automatically segmented into shots and from the content-related image segments, salient features such as region shape, intensity, color, texture and motion descriptors are extracted and used for indexing and retrieving information. In order to allow queries at a higher semantic level, some particular pictorial objects have to be detected and exploited for indexing We focus on human faces detection and recognition, given that such data are of great interest for user's queries. Here, we propose a new method for face recognition based on a wavelet packet decomposition of the face images. Each face from these wavelet coefficients which characterize the face texture, we form compact and meaningful feature vectors, using simple statistical measures. Then, we show how an efficient and reliable probalistic metric derived from the Bhattacharyya distance can be used in order to classify the face feature vectors into person classes.

The efficiency of our approach is analyzed by comparing the results with those obtained using the well-known Eigen faces method.

\section{A. Discrete Wavelet Transform}

Calculating wavelet coefficients at every possible scale is a fair amount of work, and it generates an awful lot of data. If the scales and positions are chosen based on powers of two, the so-called dyadic scales and positions, then calculating wavelet coefficients are efficient and just as accurate. This is obtained from discrete wavelet transform (DWT).

\section{B. One-Stage Filtering}

For many signals, the low-frequency content is the most important part. It is the identity of the signal. The high-frequency content, on the other hand, imparts details to the signal. In wavelet analysis, the approximations and details are obtained after filtering. The approximations are the high-scale, low frequency components of the signal. The details are the lowscale, high frequency components. The filtering process is schematically represented as in Fig. 


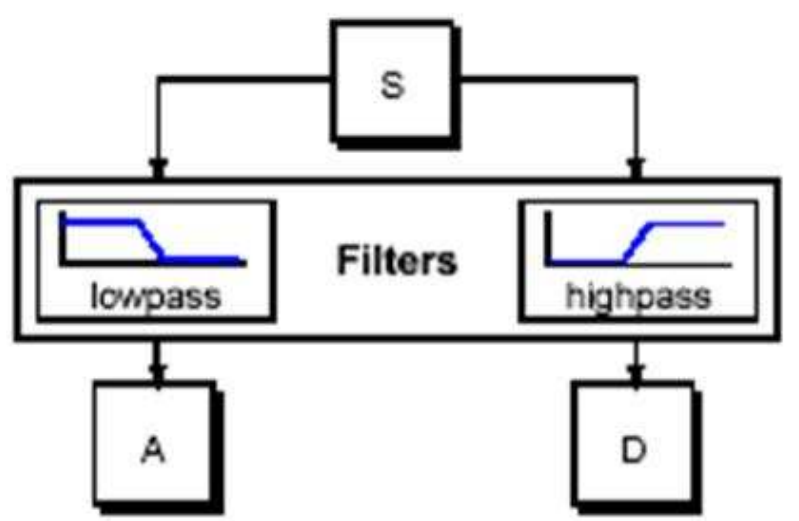

Fig. Single stage filtering

\section{Multiple-Level Decomposition}

The decomposition process can be iterated, with successive approximations being decomposed in turn, so that one signal is broken down into many lower resolution components. This is called the wavelet decomposition tree and is depicted as in Fig.

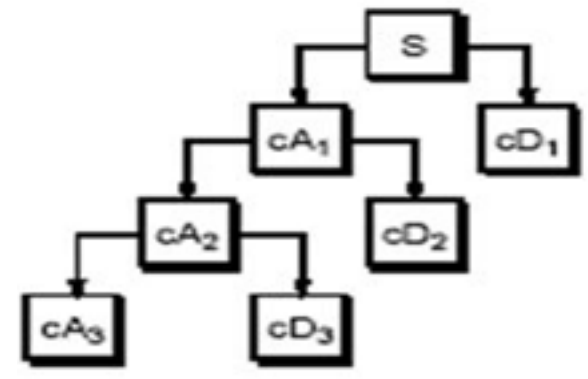

Fig. Multilevel decomposition

\section{Wavelet Reconstruction}

The reconstruction of the image is achieved by the inverse discrete wavelet transform (IDWT). The values are first up sampled and then passed to the filters. This is represented as shown in Fig.

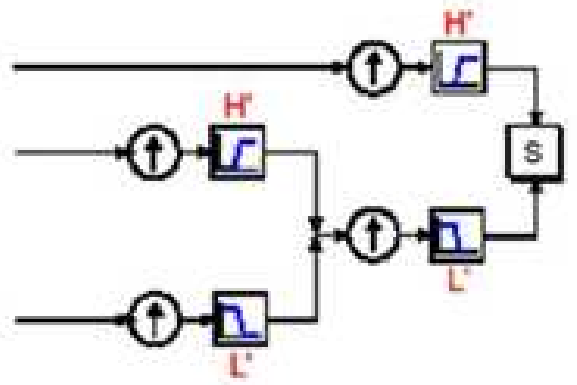

Fig. Wavelet Reconstruction

The original signal, $\mathrm{S}$, passes through two complementary filters and emerges as two signals. Unfortunately, it may result in doubling of samples and hence to avoid this, down sampling is introduced. The process on the right, which includes down sampling, produces DWT coefficients. The schematic diagram with real signals inserted is as shown in next Fig.

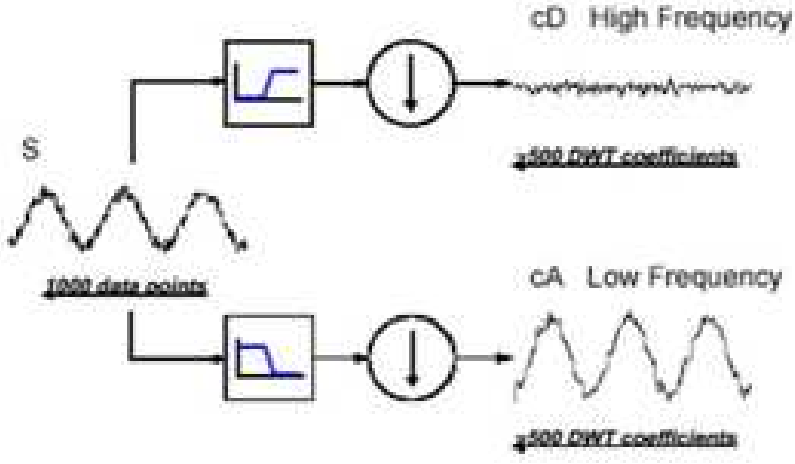

Fig. Decomposition and decimation

The wavelet analysis involves filtering and down sampling, whereas the wavelet reconstruction process consists of up sampling and filtering. Up sampling is the process of lengthening a signal component by inserting zeros between samples as shown in Fig.
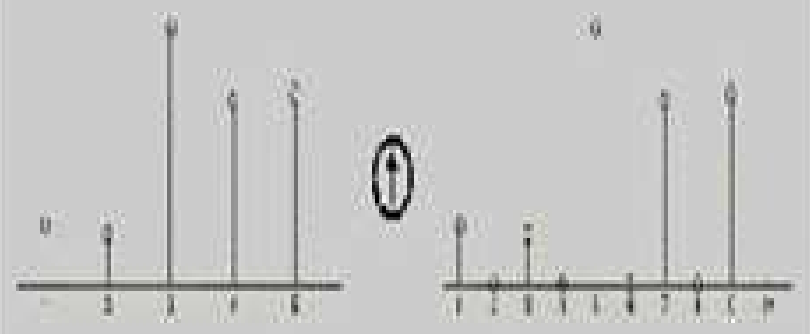

Fig. Reconstruction using up sampling Reconstructing Approximations and Details

It is possible to reconstruct the original signal from the coefficients of the approximations and details. The process yields a reconstructed approximation which has the same length as the original signal and which is a real approximation of it. The reconstructed details and approximations are true constituents of the original signal. Since details and approximations are produced by down sampling and are only half the length of the original signal they cannot be directly combined to reproduce the signal. It is necessary to reconstruct the approximations and details before combining them. The reconstructed signal is schematically represented as in.

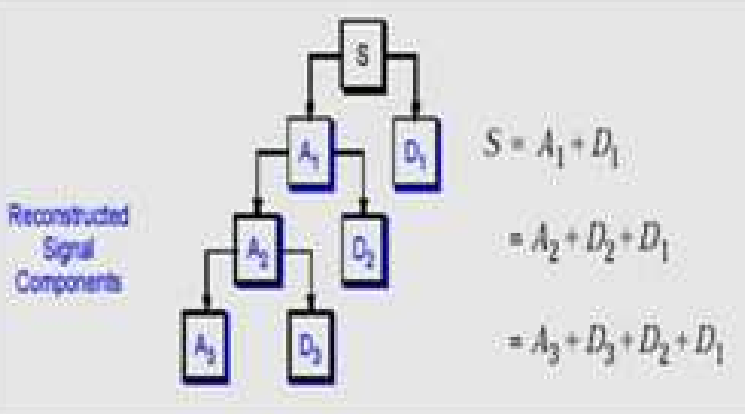

Fig. Reconstructed signal components 


\section{Wavelet Transform}

The generic form for a one-dimensional (1-D) wavelet transform is shown in Fig. Here a signal is passed through a low pass and high pass filter, $\mathrm{h}$ and $\mathrm{g}$, respectively, then down sampled by a factor of two, constituting one level of transform.

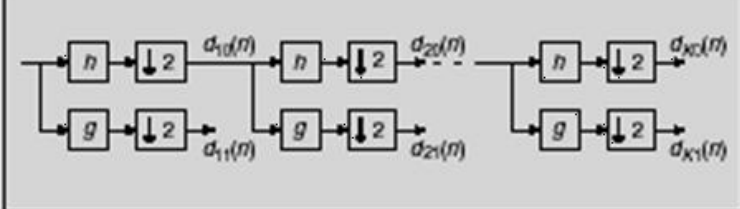

Fig. 1D Wavelet Decomposition

Repeating the filtering and decimation process on the low pass branch outputs make multiple levels or "scales" of the wavelet transform only. The process is typically carried out for a finite number of levels $\mathrm{K}$, and the resulting coefficients are called wavelet coefficients. The one-dimensional forward wavelet transform is defined by a pair of filters $s$ and $t$ that are convolved with the data at either the even or odd locations. The filters $\mathrm{s}$ and $\mathrm{t}$ used for the forward transform are called analysis filters.

$\mathrm{nH}$

$$
\mathrm{nL}
$$

$$
\text { li }=\sum_{j=-n l} s j \times 2 i+j \text { and }
$$

\section{$h i=\sum t j \times 2 i+1+j$ $\mathrm{j}=-\mathrm{nH}$}

Although land hare two separate output streams, together they have the same total number of coefficients as the original data. The output stream 1 , which is commonly referred to as the low-pass data may then have the identical process applied again repeatedly. The other output stream, h(or high-pass data), generally remains untouched. The inverse process expands the two separate low- and high-pass data streams by inserting zeros between every other sample, convolves the resulting data streams with two new synthesis filters s' and t', and adds them together to regenerate the original double size data stream.

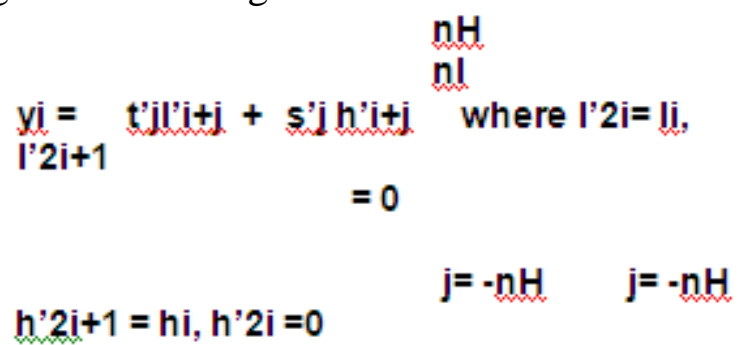

To meet the definition of a wavelet, transform, the analysis and synthesis filters $s, t, s$ ' and t' must be chosen so that the inverse transform perfectly reconstructs the original data. Since the wavelet transform maintains the same number of coefficients as the original data, the transform itself does not provide any compression. However, the structure provided by the transform and the expected values of the coefficients give a form that is much more amenable to compression than the original data. Since the filters $s, t, s^{\prime}$ and t' are chosen to be perfectly invertible, the wavelet transform itself is lossless. Later application of the quantization step will cause some data loss and can be used to control the degree of compression. The forward wavelet-based transform uses a 1-D sub band decomposition process; here a 1$\mathrm{D}$ set of samples is converted into the low-pass subband (Li) and high-pass sub band (Hi). The lowpass subband represents a down sampled lowresolution version of the original image. The highpass subband represents residual information of the original image, needed for the perfect reconstruction of the original image from the low-pass sub band.

\section{2-D Transform Hierarchy}

The 1-D wavelet transform can be extended to a twodimensional (2-D) wavelet transform using separable wavelet filters. With separable filters the 2-D transform can be computed by applying a 1-D transform to all the rows of the input, and then repeating on all of the columns.

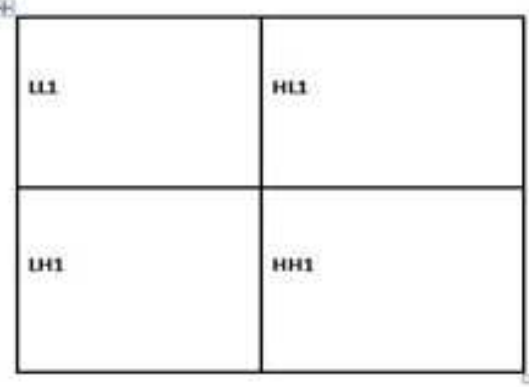

Fig. Sub band Labeling Scheme for a one level, 2-D Wavelet Transform.

The original image of a one-level $(K=1), 2-D$ wavelet transform, with corresponding notation is shown in Fig. The example is repeated for a three-level $(\mathrm{K}=3)$ wavelet expansion in Fig. In all of the discussion $K$ represents the highest level of the decomposition of the wavelet transform.

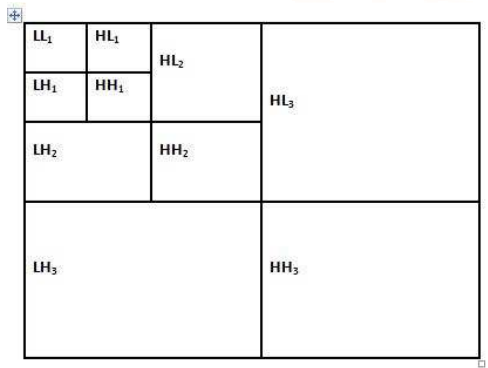

Fig. Sub band labeling Scheme for a Three Level, 2-D Wavelet Transform 
The 2-D sub band decomposition is just an extension of 1-D sub band decomposition. The entire process is carried out by executing1-Dsub band decomposition twice, first in one direction (horizontal), then in the orthogonal (vertical) direction. For example, the lowpass sub bands $(\mathrm{Li})$ resulting from the horizontal direction is further decomposed in the vertical direction, leading to $\mathrm{LLi}$ and $\mathrm{LHi}$ sub bands. Similarly, the high pass sub band (Hi) is further decomposed into $\mathrm{HLi}$ and HHi. After one level of transform, the image can be further decomposed by applying the 2-D sub band decomposition to the existing LLi sub band. This iterative process results in multiple "transform levels". In Fig. the first level of transform results in LH1, HL1, and HH1, in addition to LL1, which is further decomposed into LH2, HL2, $\mathrm{HH} 2$, LL2 at the second level, and the information of LL2 is used for the third level transform. The sub band LLi is a low-resolution sub band and high-pass sub bands $\mathrm{LHi}, \mathrm{HLi}, \mathrm{HHi}$ are horizontal, vertical, and diagonal sub band respectively since they represent the horizontal, vertical, and diagonal residual information of the original image. An example of three-level decomposition into sub bands of the image CASTLE is illustrated in Fig.

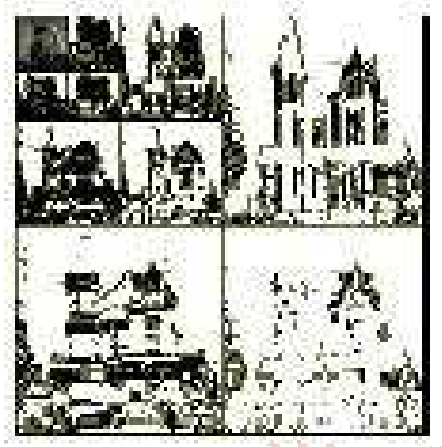

Fig. The process of 2-D wavelet transform applied through three transform level.

To obtain a two-dimensional wavelet transform, the one-dimensional transform is applied first along the rows and then along the columns to produce four sub bands: low-resolution, horizontal, vertical, and diagonal. (The vertical sub band is created by applying a horizontal high-pass, which yields vertical edges.) At each level, the wavelet transform can be reapplied to the low-resolution sub band to further decorrelate the image. Fig. illustrates the image decomposition, defining level and sub band conventions used in the AWIC algorithm. The final configuration contains a small low-resolution sub band. In addition to the various transform levels, the phrase level 0 is used to refer to the original image data.
Here we are using 2d-wavelet transformation algorithm using to get the features of the input image so that we can able to compare with database images also after this we are going to apply eigen values and vectors we are going to find the same input image and comparing with the data base images also. Finally we are going to apply Bhattacharya distance metric calculation algorithm for this to get the more and accurate image for this concept.

\section{Proposed Method}

The main characteristic of wavelets (if compared to other transformations) is the possibility to provide a multire solution analysis of the image in the form of coefficient matrices. Strong arguments for the use of multire solution decomposition can be found in psych visual research, which offers evidence that the human visual system processes the images in a multi scale way. Moreover, wavelets provide a spatial and a frequently decomposition of a image at the same time. Wavelets are also very flexible: several bases exist, and one can choose the basis which is more suitable for a given application. We think that this is still an open problem, and up to now only experimental considerations rule the choice of a wavelet form. However, the choice of an appropriate basis can be very helpful. Computational complexity of wavelets is linear with the number $(\mathrm{N})$ of computed coefficients $(\mathrm{O}(\mathrm{N}))$ while other transformations, also in their fast implementation, lead to $\mathrm{N} * \log 2(\mathrm{~N})$ complexity. Thus, wavelets are adapted also for dedicated hardware design (Discrete wavelet Transform). If the recognition task has real time computation needs, the possibility of embedding part of the process in Hardware is very interesting, like compression tasks.

Wavelet packet decomposition

The (continuous) wavelet transform of a 1-D signal $f(x)$ is defined as:

(Waf) (b)= $f \mathrm{f}(\mathrm{x}) \psi \mathrm{a}, \mathrm{b}$

(x) $d x$ $-(1)$

With $\psi a, b(x)=1 / \sqrt{ } a \psi(x-b / a)$

The mother wavelet $\psi$ has to satisfy the admissibility criterion to ensure that it is a localized zero-mean function. Equation (1) can be discretized by restraining and to a discrete lattice $(a=2 n, b € z)$. Typically, some more constraints are imposed on $\psi$ to ensure that the transform is non-redundant, complete and constitutes a multire solution representation of the original signal. This leads to an efficient real-space implementation of the transform using quadrature 
mirror filters. The extension to the 2-D case is usually performed by applying a separable filter bank to the image. Typically, a low filter and a band pass filter $(\mathrm{H}$ and $\mathrm{D}$ respectively) are used. The convolution with the low pass filter results in a so-called approximation image and the convolution with the band pass filter in a specific direction results in so-called details image. In classical wavelet decomposition, the image is split into an approximation and details images. The approximation is then split itself into a second-level approximation and details. For a -level decomposition, the signal is decomposed in the following way:

$$
\begin{aligned}
& \mathrm{An}=[\mathrm{Hx} *[\mathrm{Hy} * \mathrm{An}-1] \downarrow 2,1] \downarrow 1,2 \\
& \text { Dn, } 1=[\mathrm{Hx} *[\mathrm{~Gy} * \mathrm{An}-1] \downarrow 2,1] \downarrow 1,2 \\
& \text { Dn, } 2=\left[\mathrm{Gx}^{*}[\mathrm{Hy} * \mathrm{An}-1] \downarrow 2,1\right] \downarrow 1,2 \\
& \text { Dn, } 3=\left[\mathrm{Gx}^{*}[\mathrm{~Gy} * \mathrm{An}-1] \downarrow 2,1\right] \downarrow 1,2
\end{aligned}
$$

Where denotes the convolution operator, $\downarrow 21(\downarrow 12)$ sub-sampling along the rows (columns) and $\mathrm{A} 0=\mathrm{I}(\mathrm{x}, \mathrm{y})$ is the original image. An is obtained by low pass filtering and is the approximation image at scale $\mathrm{n}$. The details images Dni are obtained by band pass filtering in a specific direction and thus contain directional detail information at scale. The original image $I$ is thus represented by a set of sub images at several scales; $\{A n, D n i\}$. The wavelet packet decomposition that we use in our approach is a generalization of the classical wavelet decomposition that offers a richer signal analysis (discontinuity in higher derivatives, self-similarity,). In that case, the details as well as the approximations can be split. This results in a wavelet decomposition tree. Usually, an entropy-based criterion is used to select the deepest level of the tree, while keeping the meaningful information.

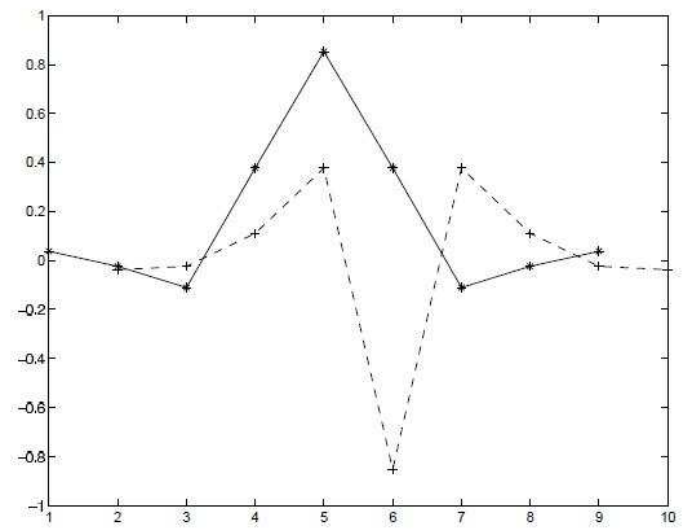

Fig: $\mathrm{H}$ (solid line) and $\mathrm{G}$ (dashed line) filters
In our experimentations, we have selected 2 levels of decomposition according to the size of the face images and we use the 16 resulting coefficient matrices which are displayed in figure. Figure 1 shows the and filters that have been applied. These filters have been selected based on trials during our experimentations. For each coefficient matrix, a set of statistical features is computed as described in the next section.

\section{Feature vectors classification}

When solving a pattern recognition problem, the ultimate objective is to design a recognition system which will classify unknown patterns with the lowest possible probability of misrecognition. In the feature space defined by a set of features $X=[x 1, \ldots \ldots \ldots, x n]$ which may belong to one of the possible $\mathrm{m}$ pattern classes, $\omega \mathrm{i}, \mathrm{i}=1, \ldots, \mathrm{m}$, an error probability can be defined but cannot be easily evaluated . Thus, a number of alternative feature evaluation criteria have been suggested in the literature. One of these criteria is based on probalistic distance measures. It is easy to show that, in the two-class case, the error probability can be written:

$$
\mathrm{e}=1 / 2\left[1-\int|\mathrm{p}(\mathrm{X} \mid \omega 1) \mathrm{P}(\omega 1)-\mathrm{p}(\mathrm{X} \mid \omega 2) \mathrm{P}(\omega 2)| \mathrm{dX}\right]
$$

According to equation (7), the error will be maximum when the integrand is zero, that is, when density functions are completely overlapping, and it will be zero when they don't overlap. The integral in (7) can be considered as the probalistic distance between the two density functions.

In our approach, the Bhattacharyya distance Bis chosen as a probalistic distance:

$B(X)=-\ln \mid \int[p(X \mid \omega 1) p(X \mid \omega 2)] 1 / 2 d X$

In the multi-classes case and to solve our problem, we make the assumption that the class-conditional probability distributions are Gaussian, that is, when the density functions are defined as:

$(\mathrm{X} \mid \omega \mathrm{i})=\left[(2 \pi) n\left|\sum \mathrm{i}\right|\right]-1 / 2 \times \exp \{-1 / 2(\mathrm{X}-\mu \mathrm{i}) \mathrm{T} \Sigma \mathrm{i}-1(\mathrm{X}-$ $\mu i)\}$

Where and are the mean vector and covariance matrix of the class distribution respectively. The multivariate integrals in the measure can be evaluated which leads to: 
$\mathrm{B}=1 / 4(\mu 2-\mu 1) \mathrm{T}[\Sigma 1+\Sigma 2]-1(\mu 2-\mu 1)+1 / 2$

in

$[|1 / 2(\Sigma 1+\Sigma 2)| / \sqrt{ }|\Sigma 1||\Sigma 2|]$

We consider that each component pair $\{\mu \mathrm{i}, \sigma \mathrm{i} 2\}$ is independent from the other component pairs of the feature vector $\mathrm{V}$. Thus, the distance between to feature vectors Vkand $\mathrm{Vl}$ is computed on a component-pair basis, that is, the distance is considered as a sum of distances relative to each of these component pairs. Using the Bhattacharrya distance, the distance Di between the component pairs of the two feature vectors $\mathrm{Vk}$ and $\mathrm{Vl}$ is:

$\operatorname{Di}(\mathrm{Vk}, \mathrm{Vl})=1 / 4(\mu \mathrm{ik}-\mu \mathrm{il}) 2 /(\sigma \mathrm{ik} 2+$ бil2)

$[1 / 2(\sigma \mathrm{ik} 2+\sigma \mathrm{il} 2) / \sqrt{ } \sigma \mathrm{ik} 2$ бil2]

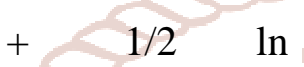

With $\forall i=1,2, \ldots, n, \mu i k=\mu i l=0$ where $n+1$ is the size of the feature vectors. As a consequence, the resulting distance D between two feature vectors Vkand Vl can be chosen as:

$\mathrm{D}(\mathrm{Vk}, \mathrm{Vl})=\Sigma \mathrm{Di}(\mathrm{Vk}, \mathrm{Vl})$

\section{Block diagram for the proposed method}

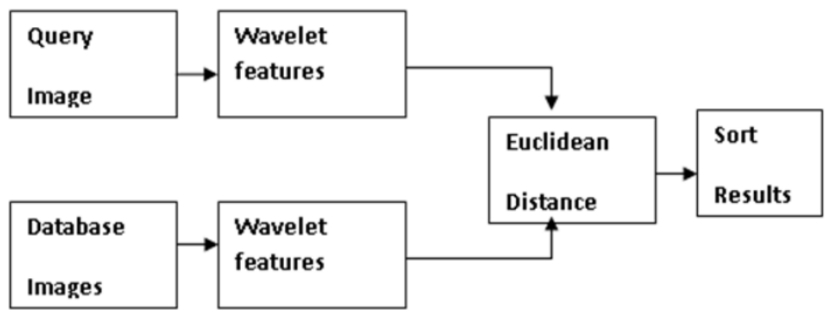

Figure: block diagram for the proposed system

Algorithm for the proposed method Input: images of the faces

Output: matching face images will be displayed

Methodology:

Step 1: [image acquisition]

Images of the faces are collected

Step 2: read the image

Step 3: wavelet decomposition of an image

Step 4: feature vector extraction

Step 5: [feature vector classification] Face feature vector classification will have done by using Bhattacharyya distance measure

Step 6: matched images will be displayed.

\section{EXPERIMENTAL RESULTS:}

In this experiment, we first extract the feature vectors of all the images in the data set and then form the mean vectors of each class (namely Vcmean), that is, we use an intra-class information. Then, we verify that each image is classified into the correct class, looking for the minimum $\mathrm{D}(\mathrm{Vk}, \mathrm{Vcmean})$ distance, for each class c. Every experiment was performed using fractions of the available images in the whole dataset. By this way, we are able to study how the size of the image dataset affects the recognition performances.

\begin{tabular}{|c|c|c|}
\hline S. no & Number of images & Recognition rate \\
\hline 1. & 10 & $100.0 \%$ \\
\hline 2. & 20 & $100.0 \%$ \\
\hline 3. & 30 & $99.6 \%$ \\
\hline 4. & 40 & $99.5 \%$ \\
\hline 5. & 50 & $99.5 \%$ \\
\hline
\end{tabular}

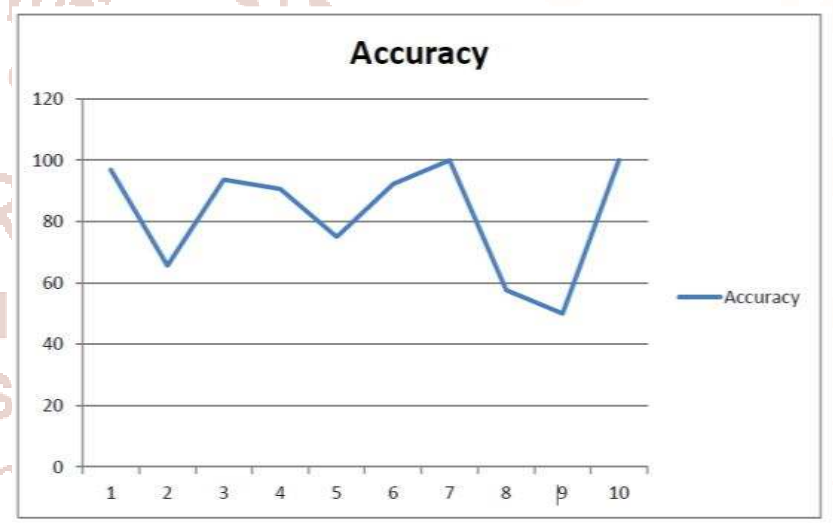

Figure: Accuracy against No of images

\section{SCREEN SHOTS}

1. Database containing collection of images

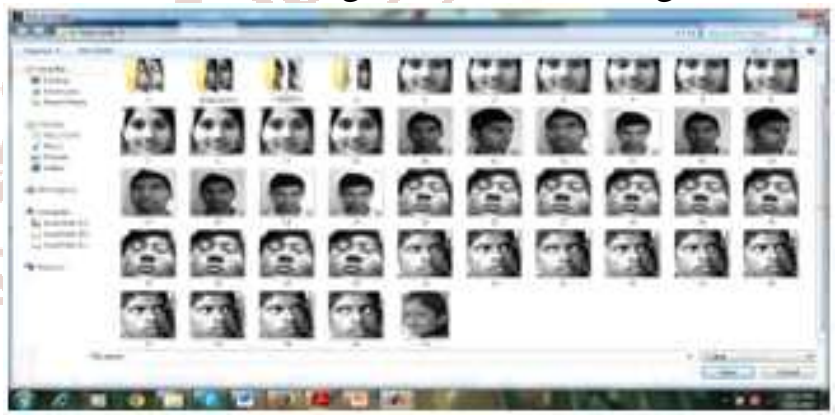

2. Initial starting window of the application through this image will be selected.

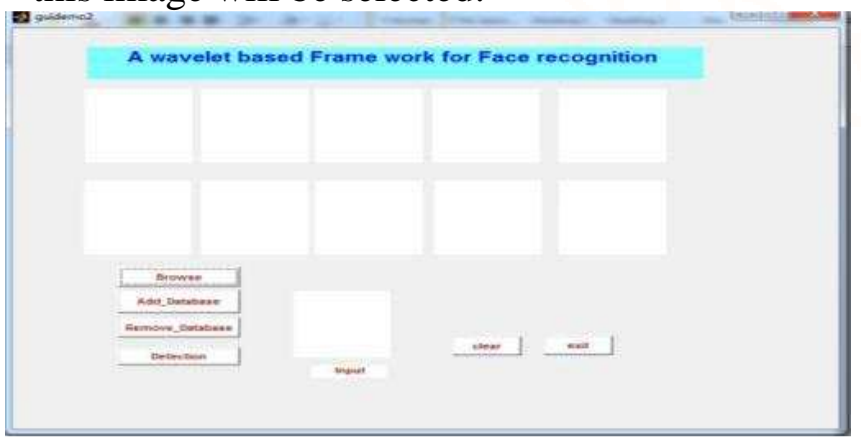


3. Initial starting window of the application through this image will be selected.

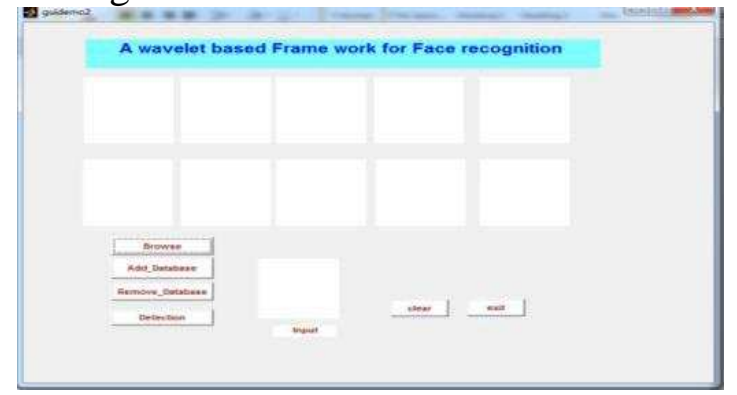

4. This window will appear after selecting an image

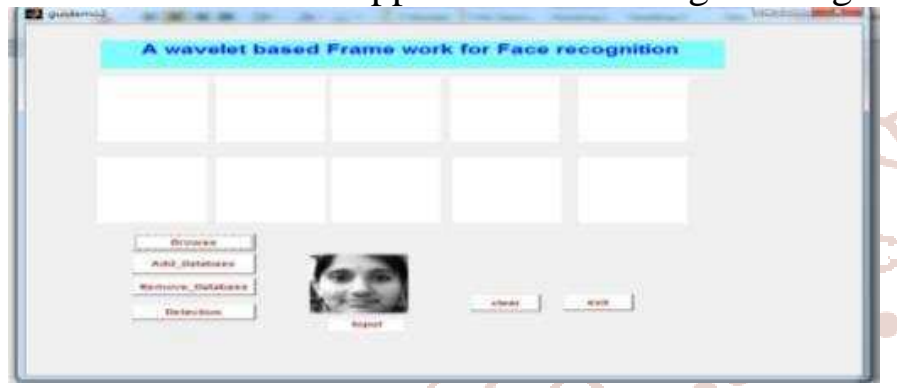

5. If image is not selected the following window will appear

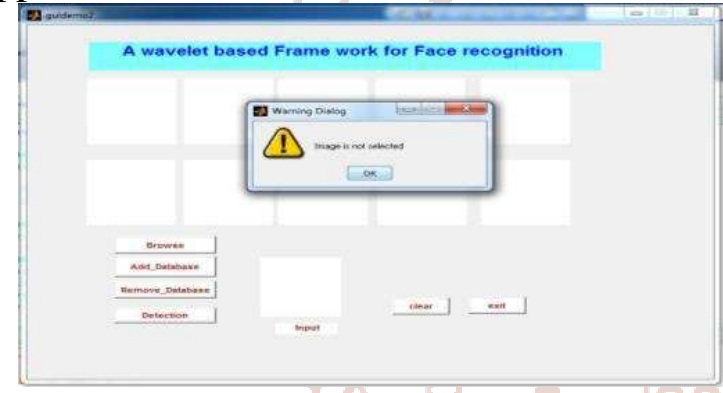

6. If the detection is successful the following window will appear

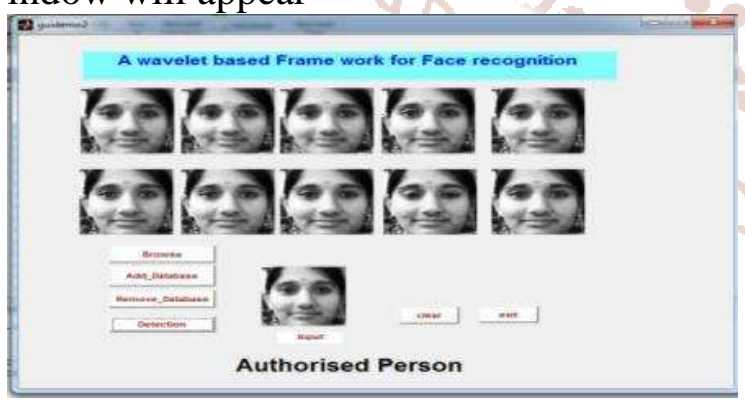

7. If the detection is not successful then the following window will appear

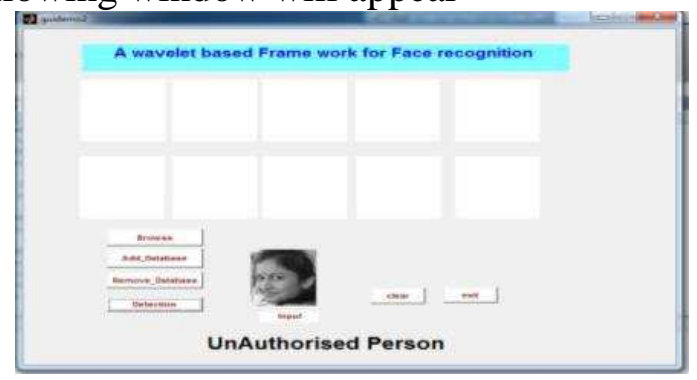

VII. CONCLUSION

Our experiments show that a small transform of the face, including translation, small rotation and illumination changes, leave the face recognition performance relatively unaffected. For both databases, good recognition rates of approximately $96.0 \%$ are obtained. Thus, the wavelet transforms proved to provide an excellent image decomposition and texture description. However, detecting features is by itself a difficult and time-consuming process so this strategy will increase the time that actually will be needed for recognition.

\section{FUTURE ENHANCEMENTS}

Some of the future works for the proposed work are Image retrieval can be done for the different shapes of vehicular object the future work can be done on the trying to recognize the gender of a person using the same algorithm.

\section{REFERENCES:}

1. P. Belhumeur, J. Hespanha, D. Kriegman. Eigen faces vs. Fisher faces: Recognition using class specific linear projection. IEEE Transactions on Pattern Analysis and Machine Intelligence, 19(7):711-720, July 1997.

2. R. Brunelli, T. Poggio. Face Recognition: Features versus Templates. IEEE Transactions on Pattern Analysis and Machine Intelligence, 11(6):10421052, 1993.

3. $=$ Y. Dai, Y. Nakano. Recognition of facial images with low resolution using a Hopfield memory model. Pattern Recognition 31(2):159-167, 1998.

4. I. Daubechies. The Wavelet Transform, TimeFrequency Localization and Signal Analysis. IEEE Transaction so Information Theory, 36(5):961- 1005, 1990. EP 24956. Esprit Project.

5. Distributed audio-Visual Archives Network. Y. $\mathrm{Fu}$. Handbook of Pattern Recognition and Image Processing. Academic Press, 1986.

6. Aziz Makandar, Anita. Patarot, Bhagirathi Halalli:" Color Image Analysis and Contrast Stretching using Histogram Equalization" International Journal of advance Information Science and Technology (IJAIST).

7. Aziz Makandar and Anita. Patarot. " Computation Pre-Processing Techniques for Image Restoration". International Journals of Computer Applications (0975-8887). Volume 113-No .4. March 2015. 
8. Aziz Makandar, Anita. Patarot, Bhagirathi Halalli," Wavelet Statistical Feature Based Malware class Recognition and Classification using Supervised Learning Classifier". Oriental Journals of computer Science \& Technology. ISSN 0974-6471 June 2017, vol.10, no. (2).

9. Gersho, R. M. Gray. Vector Quantization and Signal Compression. Kluwer Academic Publisher, 1992

10. M. Kirby, L. Sirovich. Application of the Karhunen-Loeve Procedure and the Characterization of Human Faces. IEEE Transactions on Pattern Analysis and Machine Intelligence, 12(1):103-108,
11. A. Lanitis, C. J. Taylor, T. F. Cootes. Automatic Interpretation Architectures: A system Level Review. Proc. of the 9 International Conference ICIAP'97, Vol.2, pp. 77- 84, Florence, Italy, September 1997.1

12. Aziz Makandar, Anita. Patarot, "Texture Based Malware Pattern Identification and Classification" International Journal on recent and Innovation trends I Computing and Communication Volume: 4 Issue: 6.

13. Aziz Makandar, Rashmi. S "Approaches of Pattern Recognition in analyzing the Neurological Disorders". International Conference on Cognition and Recognition (ICCR-2016). 